\title{
Isoforms of Alkaline Phosphatase Determined by Isoelectric Focusing in Patients with Chronic Liver Disorders
}

\author{
Brian H. Wallace ${ }^{1}$, John A. Lott ${ }^{1}$, John Griffiths ${ }^{2}$ and Robert B. Kirkpatrick ${ }^{3}$ \\ 1 Department of Pathology, The Ohio State University, Columbus, USA \\ 2 Department of Pathology, Medical University of South Carolina, Charleston, USA \\ 3 Department of Medicine, The Ohio State University, Columbus, USA
}

Summary: Fractionation of alkaline phosphatase isoenzymes and isoforms by isoelectric focusing is a simple procedure that resolves up to 17 fractions having alkaline phosphatase activity. The fractions are stable at $4{ }^{\circ} \mathrm{C}$, and undergo only slight changes during repeated freeze-thaw cycles. Petreatment with phospholipase-C or sialidase changes the isoelectric focusing patterns of alkaline phosphatase in serum; we recommend they not be used owing to the loss of information. We found that the alkaline phosphatase fractions provide diagnostic information in addition to that given by the common liver-function tests in patients with chronic liver diseases. Primary biliary cirrhosis and primary sclerosing cholangitis showed similar biochemical changes, but they are very different from alcoholic cirrhosis based on the common liver-function tests and the alkaline phosphatase isoform patterns obtained by isoelectric focusing. Analysis of the laboratory data using neural networks has some limited use in distinguishing chronic and chronic-active hepatitis of any cause. We have confirmed the tissue assignments made by Griffiths (Prog Clin Biochem 1989; 8:63-74) for the alkaline phosphatase fractions in liver as obtained by isoelectric focusing: Fractions $1 \mathrm{a}$ and $1 \mathrm{~b}$ show a strong correlation with biliary diseases, and fractions 2, 3, and 4 show consistent increases in patients with primary disorders of hepatocytes; these fractions have good sensitivity for hepatocyte injury, but their specificity is limited. Fraction 10 may be a marker of activated T-lymphocytes; it was abnormal in most of our patients suggesting that it is a sensitive but non-specific test. Analysis of alkaline phosphatase by isoelectric focusing deserves further evaluation, because it may facilitate the diagnosis of certain chronic liver disorders and could be a supplement to the biopsy.

\section{Introduction}

Griffiths suggested that the hepatic isoforms and band 10 of alkaline phosphatase ${ }^{1}$ ) in serum may serve as a "biochemical biopsy" in patients with liver diseases (1). Fractionating alkaline phosphatase subspecies by partial inactivation with heat, zone electrophoresis, polyacrylamide disk-gel electrophoresis, and the use of lectins to inhibit the bone fraction increases the information content of the test and may reveal the cause of an abnormality (2). Griffiths showed that isoelectric focusing separates alkaline phosphatase from serum into 17 or more fractions and proposed a system of fraction identification (1). We reported the isoelectric focusing findings in patients following orthotopic liver transplantation and found that membrane-associated-, liver-, and lipoprotein-bound alkaline phosphatase or high molecular mass alkaline

\section{1) Enzymes:}

Alanine aminotransferase, EC 2.6.1.2;

Alkaline phosphatase, EC 3.1.3.1;

Aspartate aminotransferase, EC 2.6.1.1;

Phospholipase C, phosphatidylinositol-specific, EC 3.1.4.3;

Sialidase, EC 3.2.1.18. phosphatase, are significantly higher in patients with complications as compared to those with a stable postoperative course (3). In patients with liver diseases, there is now evidence that alkaline phosphatase fractions $1 \mathrm{a}$ and $\mathrm{lb}$ reflect primary or secondary biliary ductule injury of various causes. Patients with obstructive biliary tract disease such as primary biliary cirrhosis or primary sclerosing cholangitis often have a marked increase in alkaline phosphatase fractions la and $1 \mathrm{~b}$ during the acute phase of these disorders. Increases in fraction 2 are expected in cases of hepatocyte injury of any cause, late cholestasis, other obstructive disorders, or following exposure to hepatotoxic agents. Fraction 3 is believed to originate from the reticuloendothelial cells derived from any site of origin, and fraction 4 is associated with the vascular endothelium such as capillaries and arterioles. Fractions $5,6,7,8,9$, and 11 have as yet an undefined association with liver pathology; band 5 has been reported to originate from bone $(1,4)$. Fraction 10 is found in placental trophoblasts and activated T-lymphocytes; increases in fraction 10 would be expected 
in any autoimmune proccsses or other causes of lymphocyte infiltration of the liver as is found in many recipients of transplanted livers.

\section{Goals}

We had several broad goals in this study. The most important was to identify the abnormalities of alkaline phosphatase fractions by isoelectric focusing in patients with well-defined chronic liver diseases. We included in this discovery process other commonly performed liverfunction tests in serum such as total alkaline phosphatase, alanine aminotransferase ${ }^{1}$ ), aspartate aminotransferase $^{1}$ ), and total bilirubin. Other test results such as the prothrombin time, activated partial thromboplastin time, serum albumin, serum protein electrophoresis, viral serologies, auto-antibodies, and liver biopsy results were also collected. A secondary goal was to apply statistical tools to the alkaline phosphatase fractions and liver-function tests data in an attempt to discover any relationships between chronic liver diseases and test results.

\section{Materials and Methods}

Patients and diagnoses

Patients visiting our Hepatology Clinic during a period of about 18 months and who were found clinically to have chronic liver diseases were included. The 162 unique patients were identified by one of us (RBK) for inclusion in the cohort; most of the patients had results from liver biopsies in their medical record.

\section{Laboratory tests including alkaline phosphatase} fractions by isoelectric focusing

Serum specimens, left over from routine testing of in- and outpatients, were obtained from The Ohio State University Hospitals Clinical Chemistry Laboratory. Liver-function tests and isoelectric focusing were performed immediately to minimize the possibility of enzyme loss. Alkaline phosphatase, aspartate aminotransferase, alanine aminotransferase, and total bilirubin were analyzed on a Johnson \& Johnson (Rochester, NY 14650, U.S.A.) Ektachem $700^{\circledR}$ analyzer. Isoelectric focusing of alkaline phosphatase isoforms was performed using the Resolve ${ }^{{ }_{-}}$ ALP 90-test kit, the Resolve Omega electrophoresis power supply and cells, a computerized densitometric scanner, and a 2000 volt power supply, all from Isolab, Akron, $\mathrm{OH} 44321$, USA, according to the manufacturer's directions. The $1 \%$ agarose gels were $0.85 \mathrm{~mm}$ thick and contained $25 \mathrm{ml} / 1$ ampholytes that established a $\mathrm{pH}$ gradient of 3 to 10 during electrophoresis. The application template was placed $1 \mathrm{~cm}$ toward the cathodal side from the center of the gel, and the distance between the wires that contact the filter-paper bridges in the cell was $8 \mathrm{~cm}$. We used a 75-minute electrofocusing time throughout. Minor deviations from the manufacturer's instructions are described elsewhere (3). Phospholipase $C$ and sialidase, also known as neuraminidase, were obtained in kit form from Isolab, and the manufacturer's instructions for their use were followed exactly.

\section{Results and Discussion}

Figure 1 is a schematic of high-resolution isoelectric focusing, i.e., 90 minutes of electrofocusing, and stan- dard-resolution isoelectric focusing, i. e., 75 minutes of electrofocusing. Isoelectric focusing resolves alkaline phosphatase into at least 12 distinct fractions to which specific organ sources have been assigned. In addition to the 12 fractions seen in healthy individuals, other fractions may be present in certain conditions; in pregnancy, the placental and a fetal intestinal forms are seen. In AIDS-related Kaposi's sarcoma, a characteristic abnormal fraction ("ALP-k)" is seen.

After 75 minutes of migration, alḳaline phosphatase fractions $1 \mathrm{a}$ and $\mathrm{lb}$ in normal individuals are easily discernable with alkaline phosphatase-1b usually having double the staining intensity of alkaline phosphatase-1a. Alkaline phosphatase fraction 2, which appears as three fractions (alkaline phosphatase-2a, 2b and 2c) after 90 minutes of electrofocusing, is usually a single broad band at 75 minutes. Alkaline phosphatase- 2 is one of the three most intensely-stained fractions in the alkaline phosphatase pattern of healthy adults. With the exception of alkaline phosphatase-7a and $7 \mathrm{~b}$, which form an equal-intensity doublet, alkaline phosphatase fractions 3 through 12 are singlets. Alkaline phosphatase- 5 stands out as a landmark in normals because of its intensity and the relatively large distance between it and alkaline phosphatase-4. Alkaline phosphatase- 6 lies close to alkaline phosphatase-7a, is light in staining intensity, and comprises about 2 to $3 \mathrm{U} / \mathrm{l}$ in sera from healthy individuals. Alkaline phosphatase-10 is usually one of the darkest fractions, being greater than or equal to alkaline phosphatase-2 in activity. Alkaline phosphatase- 12 has a distinctive orange-brown color that distinguishes it from other fraction; the basis of this color change is

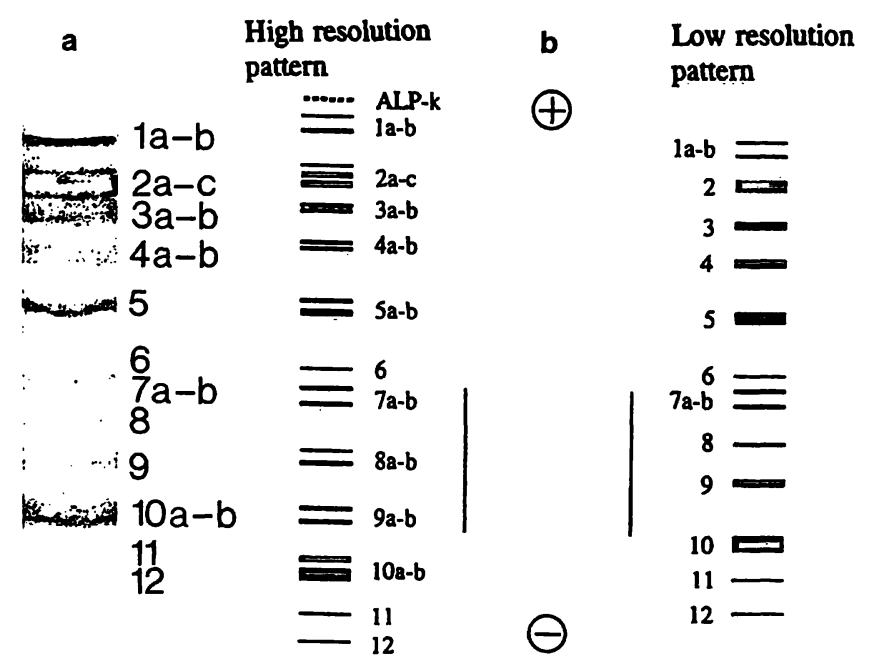

Fig. 1 Alkaline phosphatase fractionation. a) All 12 major bands and some sub-bands.

b) Schematic of the alkaline phosphatase fractions with normal, $75 \mathrm{~min}$, or high-resolution, $90 \mathrm{~min}$, isoelectric focusing. The dashed line indicates the location of the unusual fraction, named ALP-k, that was found in some individuals with AIDS-associated Kaposi's sarcoma. Lipoprotein-alkaline phosphatase, when present, appears as an outline of the application mask over alkaline phosphatase fractions 7,8, and 9; it is indicated with the vertical lines in "b.". The anode $(+)$ is at the top. 
unknown. In total, the 12 alkaline phosphatase fractions range from a pI of 3.01 to 4.86 and span a length of about $3.5 \mathrm{~cm}$ on the gel following 75 minutes of electrofocusing.

The point at which bands split with increasing electrofocusing time was determined by isoelectric focusing of the pooled serum used for run-to-run quality control in gels focused from 70 to 100 minutes in five-minutes increments. With two exceptions, each split resulted in subfractions of equal staining intensity. There was no change in the appearance in alkaline phosphatase-1a and alkaline phosphatase- $1 \mathrm{~b}$ at 90 minutes of electrofocusing. Fraction 2 divided into three bands; alkaline phosphatase-2a gave a lightly stained band while alkaline phosphatase-2b and alkaline phosphatase-2c were of equal staining intensity but darker. Alkaline phosphatase fractions $3,4,8,9,10$ and 11 split into doublets of equal staining intensity. The appearance of alkaline phosphatase- 6 , alkaline phosphatase- 7 and alkaline phosphatase12 was unchanged at 90 minutes of focusing vs. $75 \mathrm{~min}$ utes. Alkaline phosphatase-5 usually split into equal-intensity doublets; in some cases, however, alkaline phosphatase-5a and alkaline phosphatase-5b did not match in staining intensity. In these cases, there seems to be a correlation between the alkaline phosphatase- 2 activity and the appearance of a darker alkaline phosphatase- $5 \mathrm{~b}$. The reason for this finding is unknown. The utility of the high resolution pattern is uncertain because no meaning has been assigned to all the subfractions and the extent to which these subfraction may be artifactual is unknown.

\section{Electrofocusing time}

The length of electrofocusing time is an important factor in obtaining acceptable resolution. Although isoelectric focusing is by nature an end-point or concentrating technique, subtle changes in the patterns occur after the fractions have reached their isoelectric point. If the gel is properly prepared and the specimens have a low salt concentration, these changes are usually owing to cathodal endosmotic drift, termed "stretching," that has the effect of lengthening the $\mathrm{pH}$ gradient in the region of the gel where alkaline phosphatase-1 through alkaline phosphatase-12 migrate. Most of the stretching occurred in the alkaline phosphatase- 5 to alkaline phosphatase- 12 and to a lesser extent in the alkaline phosphatase-1 through alkaline phosphatase- 4 region; this is shown in figure 2. We found the optimal electrofocusing time to be about 75 minutes, and this allowed the clear separation of all 12 alkaline phosphatase fractions with some sub-fraction of alkaline phosphatase- 1 and 2.

\section{Reproducibility}

Between-run reproducibility of the alkaline phosphatase fractions determined by isoelectric focusing was as- sessed by repeatedly analyzing a pool of fresh serum having an alkaline phosphatase activity of $187 \mathrm{U} / \mathrm{l}$; the data are in table 1 . The pool had no apparent activity of alkaline phosphatase bands 11 and 12 .

\section{Specimen stability}

The between-run control demonstrated that alkaline phosphatase fractions are stable for at least one month while stored at $-20^{\circ} \mathrm{C}$. Stability at $4^{\circ} \mathrm{C}$ was tested by storing three freshly prepared serum pools having normal, slightly increased and substantially increased alkaline phosphatase activities. One aliquot from each alkaline phosphatase pool was held at $4{ }^{\circ} \mathrm{C}$ for varying numbers of days as shown in table 2 . A slight but significant upward trend was present for the lowest activity pool $(p<0.05)$. For the two higher-activity pools, the total alkaline phosphatase did not change significantly ( $p>0.05$ ) during the 32 days of storage at $4^{\circ} \mathrm{C}$. Others reported recently that alkaline phosphatase is stable at $9^{\circ} \mathrm{C}$ for at least seven days (5).

\section{Freeze-thaw stability}

The freeze-thaw stability of the total alkaline phosphatase and its isoforms was tested by subjecting two different pooled specimens to repeated cycles of freezing and thawing. A freeze-thaw cycle was defined as exposure to room temperature $\left(22^{\circ} \mathrm{C}\right.$ to $\left.26^{\circ} \mathrm{C}\right)$ for $20 \mathrm{~min}$ utes to allow complete melting of the specimen followed by storage in the freezer $\left(-20^{\circ} \mathrm{C}\right)$ for 30 minutes. As shown in table 3, 64 freeze-thaw cycles had little effect on the total alkaline phosphatase activity of the two specimens. The isoelectric focusing results shown in figure 3 demonstrate that the individual alkaline phosphatase fractions are also resistant to degradation from repeated freezing and thawing.

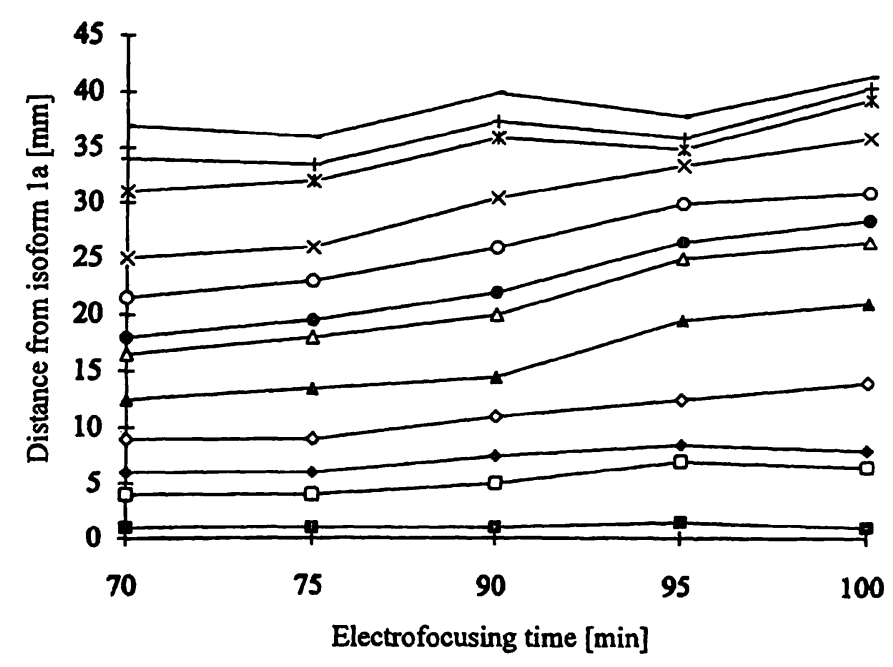

Fig. 2 Cathodal drift of alkaline phosphatase fractions with increasing electrofocusing time relative to fraction la. The alkaline phosphatase fractions from bottom to top are $1 \mathrm{~b}, 2,3,4,5,6,7$, $8,9,10,11$, and 12 . 
Tab. 1 Descriptive statistics for between-run variability of 13 replicates ${ }^{\mathbf{a}}$.

\begin{tabular}{|c|c|c|c|c|c|c|c|c|c|c|c|}
\hline & \multicolumn{11}{|c|}{ Alkaline phosphatase fraction } \\
\hline & la & lb & 2 & 3 & 4 & 5 & 6 & 7 & 8 & 9 & 10 \\
\hline $\begin{array}{l}\text { Mean } \\
\text { SD }\end{array}$ & $\begin{array}{l}1.35 \\
0.64\end{array}$ & $\begin{array}{l}4.32 \\
1.81\end{array}$ & $\begin{array}{c}22.0 \\
4.70\end{array}$ & $\begin{array}{l}8.18 \\
2.05\end{array}$ & $\begin{array}{c}12.8 \\
4.25\end{array}$ & $\begin{array}{c}15.1 \\
3.35\end{array}$ & $\begin{array}{l}2.30 \\
1.04\end{array}$ & $\begin{array}{c}13.8 \\
3.35\end{array}$ & $\begin{array}{l}7.88 \\
2.08\end{array}$ & $\begin{array}{c}17.2 \\
1.52\end{array}$ & $\begin{array}{l}77.0 \\
12.4\end{array}$ \\
\hline
\end{tabular}

a All values in $\mathrm{U} /$. The alkaline phosphatase activity of the fractions was calculated by multiplying the total activity by the percent from patients and used here had no detectable alkaline phosphaof the fraction obtained by densitometry. The pool of fresh sera

\section{Phospholipase-C and sialidase treatment of serum}

We found the best resolution of the alkaline phosphatase isoforms by working with neat, untreated serum. Phosphatidylinositol-specific phospholipase C enzymatically cleaves the membrane-bound and hepatocellular alkaline phosphatase fractions $1 \mathrm{a}, 1 \mathrm{~b}$ and 2 from the lipoproteinalkaline phosphatase complex. The lipoprotein-alkaline phosphatase fraction does not migrate during isoelectric focusing and appears as the image of the application mask that appears on the gel as a smudge over alkaline phosphatase fractions 7 through 9 . Following treatment with phospholipase-C, the staining intensity of the lipoprotein-alkaline phosphatase disappears while fraction $\mathrm{la}, 1 \mathrm{~b}$ and 2 are increased as is demonstrated in figure 4 .

Sialidase enzymatically removes the sialic acid residues attached during the post-translational modification of al-

Tab. 2 Results of alkaline phosphatase storage stability at $4{ }^{\circ} \mathrm{C}$ in fresh pooled patients' sera.

\begin{tabular}{llll}
\hline Days at $4{ }^{\circ} \mathrm{C}$ & $\begin{array}{l}\text { Specimen A } \\
\left(81 \mathrm{U} / /^{\mathrm{a}}\right)\end{array}$ & $\begin{array}{l}\text { Specimen B } \\
\left(154 \mathrm{U} / \mathrm{I}^{\mathrm{a}}\right)\end{array}$ & $\begin{array}{l}\text { Specimen C } \\
\left(618 \mathrm{U} / \mathrm{l}^{\mathrm{a}}\right)\end{array}$ \\
\hline 0 & 87 & 170 & 670 \\
2 & 89 & 162 & 679 \\
4 & 89 & 167 & 712 \\
8 & 90 & 171 & 672 \\
16 & 92 & 169 & 682 \\
32 & 94 & 176 & 723 \\
\hline
\end{tabular}

a Alkaline phosphatase activity prior to the start of the experiment. Alkaline phosphatase values in table expressed as U/I. Specimen A showed a statistically significant increase in the alkaline phosphatase with time, $p<0.05$, whereas specimens $B$ and $C$ did not, $\mathrm{p}>0.05$.

Tab. 3 Summary of data from freeze-thaw study.

\begin{tabular}{lll}
\hline Freeze-Thaw Cycles $^{\mathbf{a}}$ & $\begin{array}{l}\text { Specimen A } \\
\mathrm{U} / \mathrm{l}\end{array}$ & $\begin{array}{l}\text { Specimen B } \\
\mathrm{U} / 1\end{array}$ \\
\hline 0 (control) & 172 & 157 \\
2 & 163 & 131 \\
4 & 153 & 145 \\
8 & 153 & 134 \\
16 & 147 & 133 \\
32 & 162 & 149 \\
64 & 180 & 148 \\
\hline
\end{tabular}

\footnotetext{
a See text for description of experiment.
}

tase-11 or 12 .

kaline phosphatase. Differential sialidation of the tissue nonspecific isoforms results in differences in their size and charge and is the basis of the separation by isoelec-
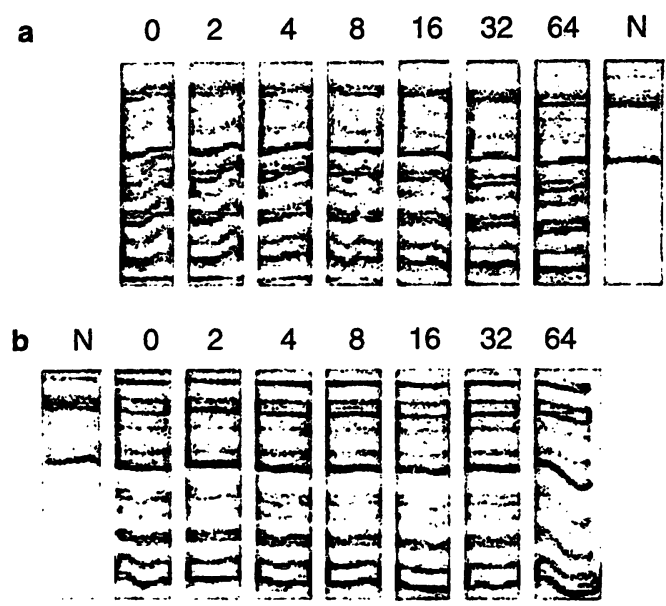

Fig. 3 Isoelectric focusing of the freeze-thaw aliquots. The upper gel, "a", and the lower gel, " $b$ ", show the results of repeated freezethaw cycles on specimen A (top) with ca. $200 \mathrm{U} / \mathrm{l}$ and B (bottom) with ca. $150 \mathrm{U} / \mathrm{l}$ alkaline phosphatase activity. From left to right, the number of cycles are $0,2,4,8,16,32$, and 64 . The right-most specimen in "a" and left-most specimen in " $b$ " is a control lane containing freshly collected serum from a healthy individual; it was not subjected to freeze-thaw cycles. The anode $(+)$ is at the top.

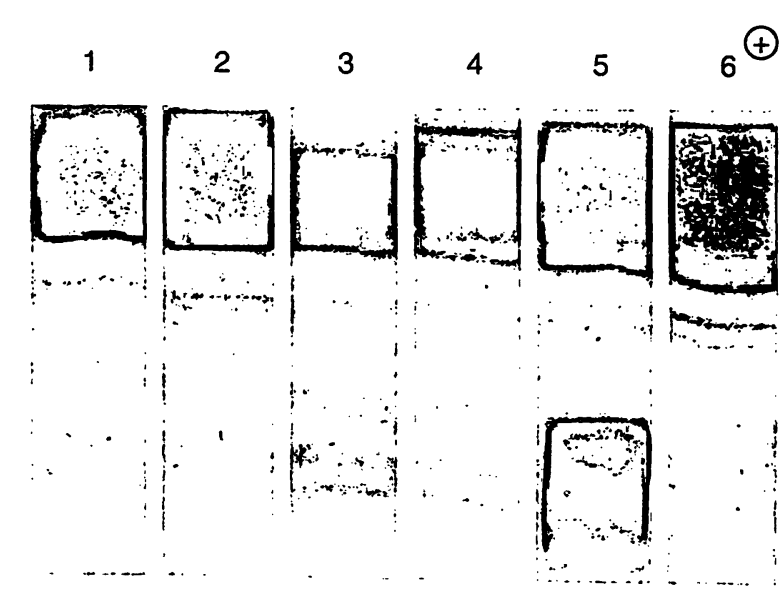

Fig. 4 Effect of phospholipase-C treatment on the lipoprotein-alkaline phosphatase complex prior to isoelectric focusing. From left to right, lanes 1, 3 and 5 are untreated specimens; lanes 1 and 3 show trace amounts of lipoprotein-bound alkaline phosphatase, and lane 5 shows a marked increase. Lanes 2,4 and 6 are replicates of lanes 1, 3 and 5 except that, prior to isoelectric focusing, they were treated with phospholipase $C$. In each of the treated specimens, no or little lipoprotein-alkaline phosphatase activity remained, while alkaline phosphatase-1a, $1 \mathrm{~b}$ and 2 were increased slightly. The anode $(+)$ is at the top. 
tric focusing. By contrast, germ cell, intestinal and placental alkaline phosphatases have different isoelectric points owing to different amino acid sequences. In theory, after the complete hydrolysis of the sialic acid residues of alkaline phosphatase with sialidase, isoelectric focusing should reveal only one fraction for the tissue non-specific forms located at the normal site for intestinal alkaline phosphatase. The position of intestinal alkaline phosphatase is unaffected, because this fraction lacks sialic acid residues and is sialidase resistant.

Sialidase treatment affected alkaline phosphatase fractions 1 to 9 as expected; however, the resultant combined fraction migrated to a position 6 to $7 \mathrm{~mm}$ cathodal to the position of the untreated intestinal alkaline phosphatase, i. e., to the position of fraction 12. Sialidase did not effect alkaline phosphatase fractions 10 or 11 that remained in the same location as the untreated specimen. Sialidase treatment shifted alkaline phosphatase12 to a position 2 to $3 \mathrm{~mm}$ towards the cathode. This phenomenon is shown in figure 5. Owing to the loss of information caused by sialidase, we elected not to use this technique.

\section{Data Analysis and Discussion}

\section{Descriptive statistics and T-test evaluation of all groups}

Means and standard deviations of the tests for the disease groupings are given in table 4 , and any statistically significant differences between the diseased and control

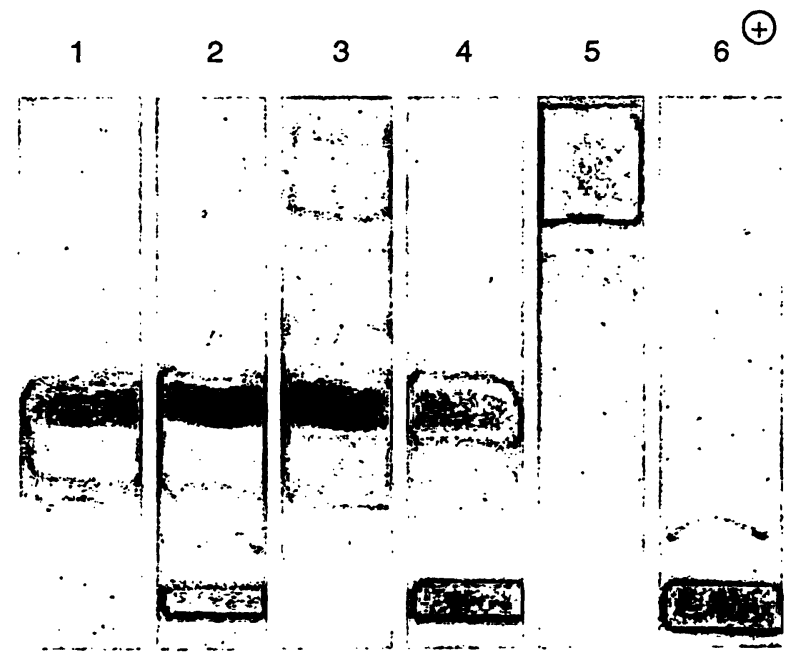

Fig. 5 Effect of sialidase ${ }^{1}$ ) treatment prior to isoelectric focusing. From left to right, lanes 1,3 and 5 are untreated specimens. Lanes 2, 4 and 6 are replicates of lanes 1,3 and 5 except that, prior to isoelectric focusing, they were treated with sialidase. The treated lane represents the enzymatic desialidation of alkaline phosphatase fractions 1 through 8 which migrate cathodal to alkaline phosphatase- 12 and are represented by the large, intensely stained fraction at the bottom of lanes 2,4 and 6 . Note that sialidase does not effect the position of alkaline phosphatase fractions 10 and 11 , while the position of alkaline phosphatase- 12 has shifted slightly towards the cathode. The anode $(+)$ is at the top. groups are given as well. Note that we divided alkaline phosphatase- 1 into fractions $1 \mathrm{a}$ and $1 \mathrm{~b}$, because we found that fraction 1 splits into two, easily resolvable fractions with the Isolab system. In patients with lesions affecting primarily the biliary tract, e.g., primary biliary cirrhosis, primary sclerosing cholangitis, and granulomatous hepatitis, we found marked increases of most of the common liver-function tests; alkaline phosphatase fractions $1 \mathrm{a}$, and (or) $1 \mathrm{~b}$ were generally much higher than those of the other liver disease groups. In patients with major hepatocyte involvement, e.g., viral, granulomatous, or autoimmune hepatitis, alkaline phosphatase-2, 3, 4 and 10 were usually increased, sometimes dramatically so. Those patients with biliary tract disorders also showed increases in alkaline phosphatase $2,3,4$, and 10; it is reasonable to conclude that these individuals had hepatocyte involvement as well in their disorder. Alkaline phosphatase-10 may be a marker of T-cell infiltration; however, we found that the test is sensitive but not specific for an autoimmune reaction.

\section{T-test evaluation of selected groups}

We chose a subset of our patients having alcoholic cirrhosis, primary biliary cirrhosis, or primary sclerosing cholangitis to determine if any of the 10 tests in table 5 could be used to discriminate between these disorders and if statistically significant findings were present. Primary biliary cirrhosis and primary sclerosing cholangitis could not be distinguished using any of the tests in table 5 with the exception of alanine aminotransferase. By contrast, most of the tests in table 5 show statistically different results $(p<0.05)$ for primary biliary cirrhosis and primary sclerosing cholangitis as compared to alcoholic cirrhosis.

Another subset of 68 patients included 43 patients with chronic hepatitis and 25 patients with chronic-active hepatitis A, B, or C. T-test comparison of patients in these two groups were made, and three tests showed significant differences: alkaline phosphatase, $p<0.001$; total bilirubin, $p<0.003$; and alkaline phosphatase- 10 , $\mathrm{p}<0.001$.

\section{Neural networks}

Kazmierczak et al. applied neural networks for the analysis of multivariate data (6). We elected to use the same approach with a neural networks program (Braincel V. 2, Promised Land Technologies, Inc., New Haven, CT 06510 USA) on a MS-DOS 486DX2 personal computer. We wanted to learn which group of tests gave the best discrimination between the 43 patients with chronic hepatitis and the 25 with chronicactive viral hepatitis. We chose these two groups owing to the similarity of the disorders and because we 


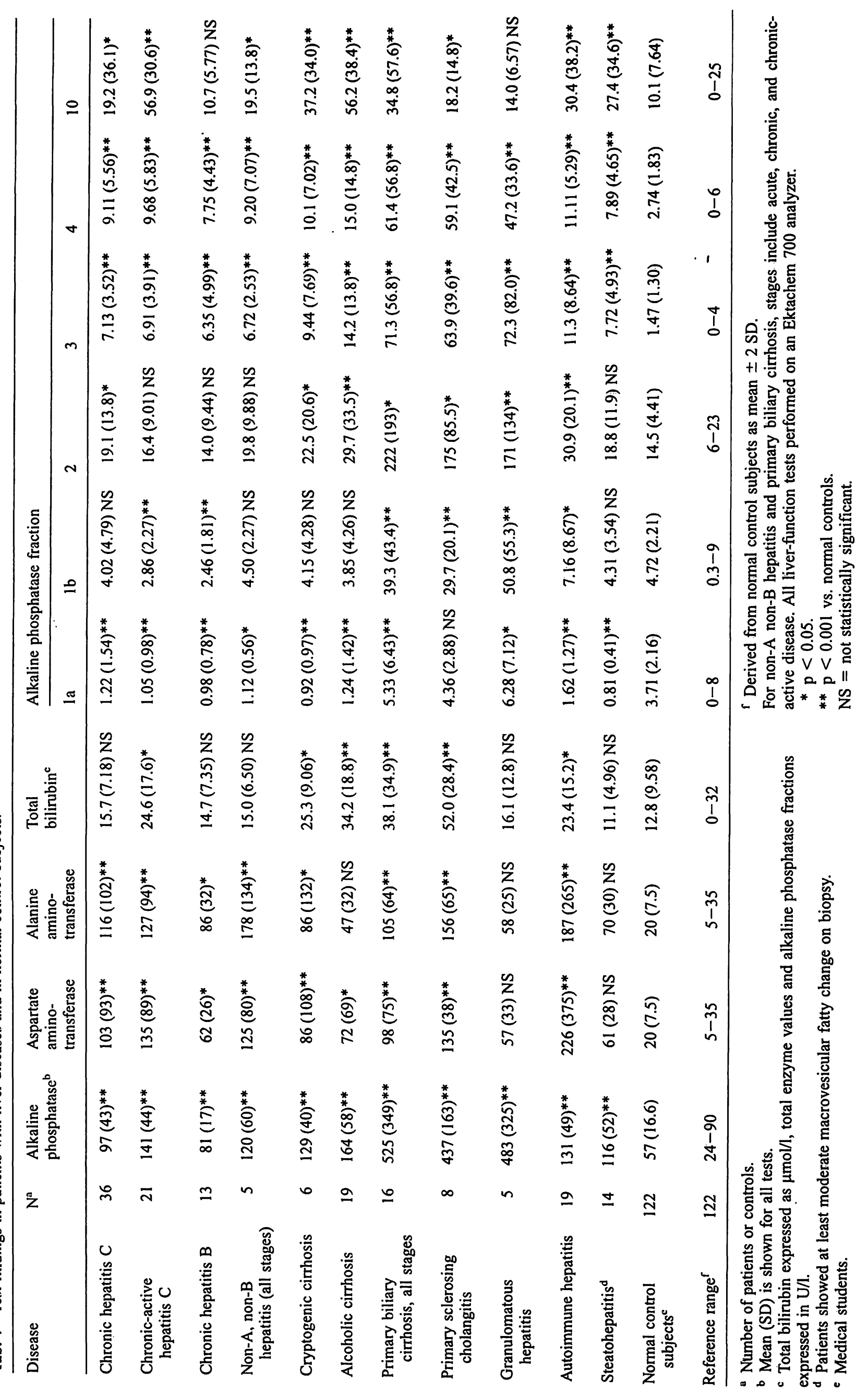


Tab. 5 Correlation of laboratory tests in patients with three types of cirrhosis.

$$
\text { Alcoholic cirrhosis }
$$

\section{Alcoholic cirrhosis}

Primary biliary cirrhosis

Primary sclerosing cholangitis
Alkaline phosphatase, alanine aminotransferase, aspartate aminotransferase, alkaline phosphatase- $1 \mathrm{a}, 1 \mathrm{~b}, 2,3,4$

Alkaline phosphatase, alanine aminotransferase, aspartate aminotransferase, alkaline phosphatase-1a, 1b, 2, 3, 4, 10
$-$

Alanine aminotransferase
Tests shown had $p<0.05$ in comparing any two disease groups. For example, in comparing primary biliary cirrhosis and alcoholic cirrhosis, alkaline phosphatase, alanine aminotransferase, aspartate aminotransferase, alkaline phosphatase-la, 1b, 2, 3, and 4 were significantly different in patients with these diagnoses. had data from a fairly large number of patients. We used 34 of these subjects, randomly drawn, as the training set for the neural networks "expert." Briefly, the neural networks expert was trained so that a case of chronic-active hepatitis would predict a " 0 " as the output neuron, and chronic hepatitis would predict a " 1 " as the output neuron. These outcome assignments were made by us and are arbitrary; however, integer values are usually assigned to output neurons. We trained the expert to a $1 \%$ error rate; in all cases, the training time on the personal computer was $<5 \mathrm{~min}$.

In four separate trials, the training input neurons were:

1. Serum alkaline phosphatase, alanine aminotransferase, aspartate aminotransferase and total bilirubin;

2. Serum alkaline phosphatase fractions $1 \mathrm{a}, 1 \mathrm{~b}, 2,3,4$, 5 , and 10 ;

\section{All 11 of the tests in trials 1 and 2; and}

4. Alkaline phosphatase, total bilirubin, and alkaline phosphatase-10. Having now trained the expert, we calculated the output neuron values for the above four trials in the remaining 34 patients; these of course were not part of the training set. The success of the expert in predicting the correct diagnosis is shown in table 6. Output neuron values of \pm 0.2 of the expected values, i. e., 0 to 0.2 for chronic-active hepatitis and 0.8 to 1.2 for chronic hepatitis, were called "correct." The best combi- nation of tests for discriminating between chronic and chronic-active hepatitis was alkaline phosphatase, alkaline phosphatase-10, and total bilirubin. The other combinations of tests were only slightly inferior. For the diagnosis of chronic-activity hepatitis, the accuracy of the neural networks output were practically the same for the four test groups.

We also prepared receiver operating characteristic curves (ROC) as was done by others (7) for the above combinations of tests. The areas under the curves for the four cases were not statistically different and were in the range of 0.87 and 0.92 . Several patients with a diagnosis of chronic or chronic-active hepatitis were outliers on two or more of the four sets of test combinations suggesting the possibility of an incorrect classification.

\section{Disease Staging with Alkaline Phosphatase by Isoelectric Focusing}

Patients with primary biliary cirrhosis (8) demonstrated a consistent increase for alanine aminotransferase, aspartate aminotransferase, alkaline phosphatase and total bilirubin regardless of the stage of the disease. Alkaline phosphatase was increased in all of the 16 cases, ranging from mild, i.e., 1.1 times the upper reference limit, to grossly increased, i. e., 12.6 times the upper reference limit. Alanine aminotransferase, aspartate aminotransferase and total bilirubin were increased in most

Tab. 6 Ability of neural networks to distinguish chronic-active from chronic hepatitis.

\begin{tabular}{lll}
\hline Tests in group & $\begin{array}{l}\text { Chronic-active hepatitis, } \\
\text { correct assignment }^{\mathrm{a}}\end{array}$ & $\begin{array}{l}\text { Chronic hepatitis, } \\
\text { correct assignment }^{\mathrm{a}}\end{array}$ \\
\hline $\begin{array}{l}\text { Alkaline phosphatase, aspartate aminotransferase, alanine aminotransferase, } \\
\text { total bilirubin }\end{array}$ & $83 \%^{\mathrm{b}}$ & $77 \%$ \\
Alkäline phosphatase-1a, 1b, 2, 3,4,10 & $75 \%$ & $68 \%$ \\
All in above two groups & $83 \%$ & $77 \%$ \\
Alkaline phosphatase, alkaline phosphatase fraction 10, total bilirubin & $75 \%$ & $82 \%$ \\
\hline
\end{tabular}

a Patients were in "predict" output group, not in "training" group. There were 12 patients in chronic-active hepatitis predict group, and 22 in chronic hepatitis predict group. b A "correct" diagnosis was defined as having a neural network predict output between 0 and 0.2 for chronic-active hepatitis or an output between 0.8 and 1.2 for chronic hepatitis. 
cases with the average increase being about twice the upper reference limit. We found no relationship between the activity of alanine aminotransferase and aspartate aminotransferase and disease stage, whereas total alkaline phosphatase and total bilirubin showed a correlation in stages I through III but not in stage IV.

Alkaline phosphatase fractions $1 \mathrm{a}, 1 \mathrm{~b}, 2,3,4$ and 10 suggest the biochemical derangement that corresponds to the underlying pathology and histological characteristic of each stage of primary biliary cirrhosis. In stage I, there is random, florid, focal destruction of the septal and interlobular bile ducts that are surrounded by a dense infiltrate of lymphocytes, both helper and suppressor populations. The inflammatory reaction is largely confined to the portal triads. Cholestasis begins to appear at this stage. Alkaline phosphatase fractions confirm the florid duct lesions with increase in the liver cell fractions alkaline phosphatase-2, 3, and 4. A mild increase in alkaline phosphatase-10 was consistent with a focal-T-cell infiltrate.

In stage II the ductal involvement is more global, with a reduction in the number of normal bile ducts and their replacement with bizarre forms. The inflammatory infiltrate extends beyond the portal triads into the surrounding parenchyma with destruction of some cells in the limiting plates. In the periportal regions, ductular proliferation creates tangled knots and strands of epithelium devoid of lumina. Cholestasis is more evident during this stage. The increase in alkaline phosphatase-2, 3, and 4 in stage II disease corresponds to the global ductal involvement and increasing cholestasis. The presence of increased alkaline phosphatase- 10 is indicative of the increase in both the number and distribution of the chronic T-cell infiltrates.

In stage III, the inflammatory infiltrate is less prominent and is replaced by fibrous septa that may interconnect portal areas to create micronodules. There is a distinct reduction in the number of bile ducts. The cholestasis at this stage is more marked than in stages I and II. The profound cholestasis is apparent in the striking increase in liver cell alkaline phosphatase-2, 3, and 4. A reduction in the alkaline phosphatase- 10 activity probably reflects the less prominent nature of the inflammatory cell infiltrate.

In stage IV the fibrous septa have uniformly bridged the portal areas enclosing individual lobules creating overt micronodular cirrhosis. There is a scant lymphocytic infiltrate within the fibrous strands. Larger bile ducts have virtually disappeared and cholestasis is severe. The increase in alkaline phosphatase-2, 3, and 4 and the decrease of alkaline phosphatase- 10 reflects the cirrhotic state and the scant chronic cell infiltrates, respectively. Figure 6 shows the distinct alkaline phosphatase isoform pattern for each stage of primary biliary cirrhosis.

\section{Primary sclerosing cholangitis}

Primary sclerosing cholangitis shows a moderate to substantial increase in liver-function tests in nearly all cases, a pattern similar to that seen in primary biliary cirrhosis. Alkaline phosphatase is four times the upper reference limit. This is illustrated in table 4 . Primary biliary cirrhosis is marked by a segmental, random and uneven chronic fibrosing inflammatory reaction involving single or varying combinations of the extrahepatic and intrahepatic bile ducts, and less çommonly the pancreatic duct. The intrahepatic ductal lumina may be entirely obliterated leaving only a fibrous scar; in the regions proximal to the narrowing, there may be ductal dilatation. A mononuclear inflammatory infiltrate is accompanied by occasional neutrophils and eosinophils. The marked increase in the liver cell fractions, alkaline phosphatase-1, 2, 3, and 4 correlates with the chronic fibrosing condition. Although mild, the average increase in alkaline phosphatase-10 corresponds to the chronic inflammatory infiltrate.

\section{Granulomatous hepatitis}

All patients with granulomatous hepatitis had an increased alkaline phosphatase ranging from a slight increase in one case to between three- and eight-times the upper reference limit in the remaining cases. With the exception of one patient who had slightly increased transaminases and total bilirubin, all patients had normal values for alanine aminotransferase, aspartate aminotransferase and alkaline phosphatase. Isoelectric focusing showed a consistent and distinctive pattern of grossly increased fractions $1 \mathrm{a}, 1 \mathrm{~b}, 2,3$, and 4 , while alkaline phosphatase- 10 was within the upper reference limit or slightly increased. The lipoprotein-alkaline phosphatase fraction was present in every case; the significance of this is unknown. In the single case with

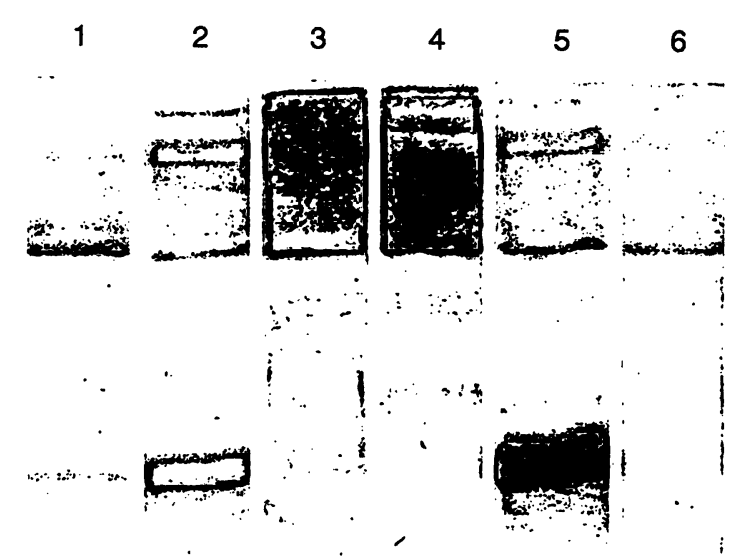

Fig. 6 Isoelectric focusing patterns of four stages of primary biliary cirrhosis. Left to rigth, lane 1 , stage $I$, alkaline phosphatase $135 \mathrm{U} / \mathrm{l}$; lane 2, stage II, alkaline phosphatase $165 \mathrm{U} / \mathrm{f}$; lane 3, stage III, alkaline phosphatase $685 \mathrm{U} / \mathrm{l}$; lane 4, stage IV, alkaline phosphatase $774 \mathrm{U} / /$; lane 5 , end-stage primary biliary cirrhosis, alkaline phosphatase $397 \mathrm{U} / \mathrm{l}$; lane 6, normal control, alkaline phosphatase $58 \mathrm{U} / \mathrm{l}$. The anode $(+)$ is at the top. 
increased alanine aminotransferase, aspartate aminotransferase and total bilirubin, the medical record indicated that the patient also had "mild Crohn's disease" and esophageal varices. A possible explanation for this finding is presented by Greenstein (9) who found that in about 5\% of cases of Crohn's disease and ulcerative colitis, there was concomitant hepatocellular disease.

\section{Autoimmune hepatitis}

As a group, patients with autoimmune hepatitis showed variable isoelectric focusing results. However, when categorized by their disease stage, either chronic or chronic-active, a consensus was noted between their clinical and isoelectric focusing data. Patients in the chronic subgroup showed within-upper reference limit to mildly increased alkaline phosphatase values. A more prominent, although variable, increase was noted for alanine aminotransferase, aspartate aminotransferase and total bilirubin with transaminases ranging from within reference to seven times the upper reference limit. The patients with increased total bilirubin were also those with increased transaminases. Marked increases were present for fractions 2, 3, 4, and 10; the latter suggests a lymphocyte infiltrate.

Patients in the chronic-active hepatitis subgroup showed a different pattern of results than those in the chronic subgroup. Alkaline phosphatase activity was increased in all cases to roughly twice the upper reference limit. Transaminases were extremely variable, ranging from within reference in about one-half of the patients, to slightly increased in one quarter, to grossly increased in the remaining patients. The maximum alanine aminotransferase and aspartate aminotransferase values, $925 \mathrm{U} / \mathrm{l}$ and $1416 \mathrm{U} / \mathrm{l}$, respectively, were found in this subgroup.

Autoimmune hepatitis has the morphologic appearance of viral hepatitis, and as such is characterized in the chronic stage as mild inflammation of the portal triads. The chronic-active stage is characterized by extensive portal inflammation that spills out of the portal tracts into the periportal parenchyma by piecemeal bridging necrosis. The isoelectric focusing data supports the histologic findings for these two stages. Chronic autoimmune hepatitis has an alkaline phosphatase pattern that is similar to chronic hepatitis $C$, except that alkaline phosphatase- 2 is increased to a greater degree in the au-

\section{References}

1. Griffiths J. Enzymatic profiles of hepatic disease. Prog Clin Biochem 1989; 8:63-74.

2. Nemesánszky E. Alkaline phosphatase. In: Lott JA, Wolf PL, editors. Clinical enzymology, a case-oriented approach. Chicago, London: Year Book Medical Publishers, 1986:47-73.

3. Randolph-Habecker J, Lott JA, Tesi RJ. Alkaline phosphatase isoforms in serum after liver allograft surgery. Clin Chem 1994; 40:1271-7. toimmune disease. The pattern of chronic-active autoimmune hepatitis is also similar to that of chronic-active hepatitis $\mathrm{C}$ except that alkaline phosphatase fractions $1 \mathrm{a}$, $1 \mathrm{~b}, 2$ and 3 are more prominent in the autoimmune disease, while alkaline phosphatase-10 is more promiment in the viral disease.

\section{Steatohepatitis}

The patients in the steatohepatitis group had liver diseases of varying origin. Despite the variety of diseases that are present, they share the common histological finding of moderate macrovesicular fatty change. Alanine aminotransferase, aspartate aminotransferase, alkaline phosphatase and total bilirubin are increased in about half of the patients with increases limited to $<2$ times the upper reference limit for each test. Total bilirubin was within the upper reference limit for all cases.

\section{Conclusions}

Isoelectric focusing of the multiple molecular forms of alkaline phosphatase is a simple technique and is no more difficult than ordinary protein electrophoresis (10). The isoelectric focusing method described here gives more information than heat inactivation or ordinary electrophoresis (11) and is superior to older isoelectric focusing techniques described elsewhere $(12,13)$. Griffith's assignment of alkaline phosphatase fractions la and $1 \mathrm{~b}$ as having a duct or ductule origin was confirmed by our finding of marked increases of these fractions in patients with primary biliary cirrhosis, primary sclerosing cholangitis, and granulomatous hepatitis. Alkaline phosphatase $1 \mathrm{~b}$ was also increased in most of the patients with autoimmune disorders; $1 \mathrm{a}$ and $1 \mathrm{~b}$ were normal or only slighly increased in those with viral hepatitis, alcoholic cirrhosis or steatohepatitis. Fractions 2, 3, and 4 that are believed to reflect hepatocyte injury are sensitive but non-specific markers; they were abnormally increased in nearly all of our patients with chronic liver diseases. Alkaline phosphatase fraction 10, is a sensitive but non-specific test for lymphocyte infiltration; it showed the highest values in chronic-active hepatitis, especially in hepatitis $\mathrm{C}$, cryptogenic cirrhosis, alcoholic cirrhosis, primary biliary cirrhosis, primary sclerosing cholangitis, and autoimmune hepatitis.

4. Griffiths J, Vernocchi A, Simoni E. Transient hyperphosphatasemia of infancy and childhood. A study of serum alkaline phosphatase by electrofocusing techniques. Arch Pathol Lab Med 1995; 119:784-9.

5. Heins M, Heil W, Withold W. Storage of serum or whole blood samples? Effects of time and temperature on 22 serum analytes. Eur J Clin Chem Clin Biochem 1995; 33:231-8. 
6. Kazmierczak SC, Catrou PG, Van Lente F. Diagnostic accuracy of pancreatic enzymes evaluated by use of multivariate data analysis. Clin Chem 1993; 39:1960-5.

7. Zweig $\mathrm{MH}$, Campbell G. Receiver-operating characteristic (ROC) plots: a fundamental evaluation tool in clinical medicine. Clin Chem 1993; 39:561 - 77.

8. Cotran RS, Kumar V, Robbins SL.Pathologic basis of disease. 5th ed. Philadelphia: WB Saunders, 1994:831-96.

9. Greenstein AJ, Janowitz HD, Sachar DB. The extra-intestinal manifestations of Crohn's disease and ulcerative colitis: a study of 700 patients. Medicine 1976; 55:401-12.

10. Griffiths J, Black J. Separation and identification of alkaline phosphatase isoenzymes and isoforms in serum of healthy persons by isoelectric focusing. Clin Chem 1987; 33:2171-7.

11. Price CP. Multiple forms of human serum alkaline phosphatase: detection and quantitation. Ann Clin Biochem 1993; 30:355-72.
12. Sinha PK, Bosisio-Bianchi A, Meyer-Sabellek W, Righetti PG. Resolution of alkaline phosphatase isoenzymes in serum by isoelectric focusing in immobilized $\mathrm{pH}$ gradients. Clin Chem 1986; 32:1264-8.

13. Sanderink G-J, Artur Y, Paille F, Galteau M-M, Siest G. Microscale two-dimensional electrophoresis of alkaline phosphatase from serum. Clin Chem 1988; 34:730-5.

Received April 10/July 5, 1996

Corresponding author: Prof. John A. Lott, Ph. D., Department of Pathology, The Ohio State University Medical Center, Starling Loving M-368, Columbus, Ohio 43210, USA 